\title{
Jacques Arènes, Croire au temps du Dieu fragile. Psychanalyse du deuil de Dieu
}

Paris, Éditions du Cerf, coll. « Sciences humaines et religions », 2012, $394 \mathrm{p}$.

\section{Daniel Vidal}

\section{(2) OpenEdition}

\section{Journals}

Édition électronique

URL : http://journals.openedition.org/assr/24233

DOI : $10.4000 /$ assr.24233

ISSN : $1777-5825$

Éditeur

Éditions de l'EHESS

Édition imprimée

Date de publication : 30 décembre 2012

Pagination : 113

ISSN : 0335-5985

\section{Référence électronique}

Daniel Vidal, « Jacques Arènes, Croire au temps du Dieu fragile. Psychanalyse du deuil de Dieu », Archives de sciences sociales des religions [En ligne], 160 | octobre-décembre 2012, mis en ligne le 21 février 2013, consulté le 21 septembre 2020. URL : http://journals.openedition.org/assr/24233; DOI : https://doi.org/10.4000/assr.24233

Ce document a été généré automatiquement le 21 septembre 2020.

(c) Archives de sciences sociales des religions 


\section{Jacques Arènes, Croire au temps du Dieu fragile. Psychanalyse du deuil de Dieu}

Paris, Éditions du Cerf, coll. « Sciences humaines et religions », 2012, $394 \mathrm{p}$.

Daniel Vidal

\section{RÉFÉRENCE}

Jacques Arènes, Croire au temps du Dieu fragile. Psychanalyse du deuil de Dieu, Paris, Éditions du Cerf, coll. « Sciences humaines et religions », 2012, 394 p. 
Si « la mort de Dieu», ou «la sortie du religieux ", ces emblèmes du désenchantement moderne, imposent de penser en termes radicalement nouveaux l'homme face à un monde délesté de toute transcendance, qu'en doit-il aller aujourd'hui de la question centrale du sujet? Telle est l'interrogation de Jacques Arènes, qui sollicite la psychanalyse pour tenter de saisir au cœur du psychisme, les instances et opérations qui vont permettre que se fasse le « deuil de Dieu » en assurant, en cette perte, la possibilité d'un gain. Un ouvrage précédent - La quête spirituelle hier et aujourd'hui. Un point de vue psychanalytique, Le Cerf, 2011 traitait déjà de cette question, mais sans la confronter aussi directement à l'effondrement des garants métasociaux de l'acte de croire. Le présent ouvrage se fonde sur un coup de force, qui en fait

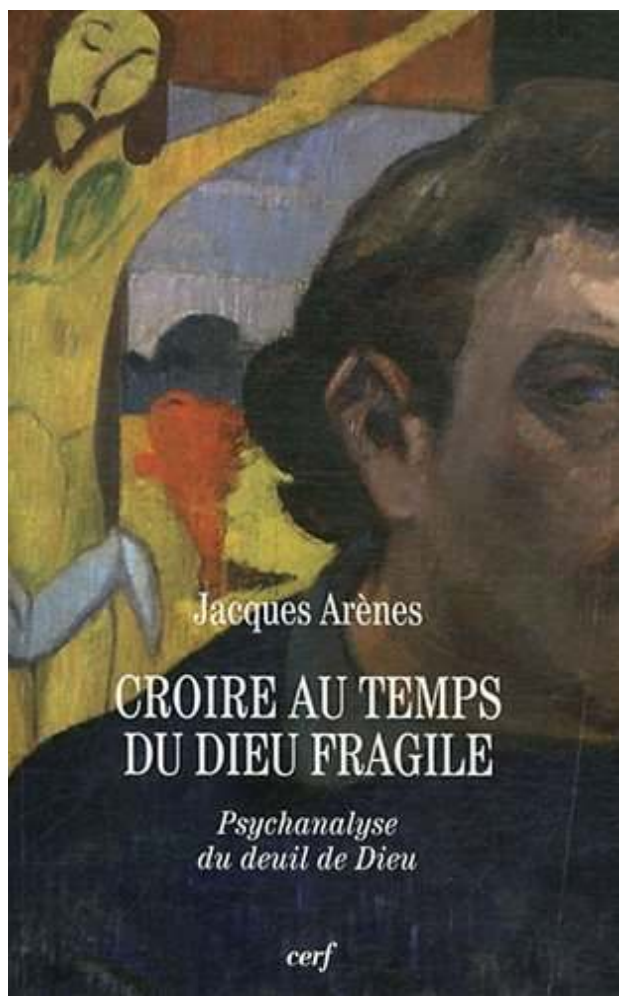
tout l'intérêt, et la complexité. Paradoxe, en effet, que cette question du sujet, si l'on pose avec l'auteur, dans la stricte observance des thèses de Marcel Gauchet, que cette impossibilité d'imaginer aujourd'hui « tout fondement transcendant à la culture » est cela même qui autorise, de ce sujet, la naissance plénière. Le sujet au principe du désenchantement, et en son issue. Venu à son autonomie et à sa capacité de demeurer au vif d'une détresse et d'une absence. D'un trauma. La subjectivation est précisément cet opérateur qui est comptable de l'exil à jamais de Dieu, en même temps qu'elle en est la conséquence. L'équation a été de longue date posée, entre l'excès de Dieu et sa perte, entre la raison mystique et l'invention du sujet, entre l'anéantissement en Dieu et l'institution de la subjectivité. Entre perte de ce que la mystique du xviie siècle, pour dire Dieu, nommait Objet, et ce que la même mystique nommait l'intérieur ou fond de l'âme. Sans doute l'auteur ne méconnaît-il pas ces conjonctions qui confortent les assises de son argumentation : si bien que, dans cet effacement du "divin », le travail de deuil à quoi tout «croyant »- et, si l'on peut dire, bien au-delà, tout profane -, est requis de se confronter, est du même coup travail du sujet.

La spiritualité du "siècle des saints » disait qu'un " espace intérieur » se disposait en l'homme pour y accueillir son Dieu. C'est aujourd'hui pour y « rapatrier la complexité de la réalité extérieure » qu'un tel «espace» est revendiqué. Signe des temps : la « mort de Dieu » est proclamée, Nietzsche, bien sûr, mais dans l'héritage des Lumières, et au fondement de la pensée moderne. Dès ces Lumières, elles-mêmes venues de Descartes et Spinoza, au temps même où s'opère la sortie de religion, se mettent en place les cadres conceptuels qui vont permettre de dire le sujet, sa conscience, et, de celle-ci, les instances exactes. Dont l'Inconscient, que Jacques Arènes, fort justement, dispose dans un long héritage que l'on a parfois contesté. Pour l'auteur, la psychanalyse, parce qu'elle «modélise un monde interne exempt de hasard, mais lieu 
de la complexité ", s'avère l'outil le plus pertinent pour dire la catastrophe et les moyens de la conjurer, pour dire le trauma, sa traversée, et ses issues. Cet accomplissement du sujet - dans le moment même où il s'éprouve en perte de Dieu et traverse cette absence radicale comme risque de déperdition irréversible de sens, mais chance aussi bien de recommencement -, ne se peut que comme aussitôt « gisement d'altérité »: comme inscription de l'autre en tant que cet autre serait le tenant-lieu symbolique d'un dieu failli. Le sujet ne s'institue que sous condition de cette injonction d'être toujours en lui-même cet autre. Le sujet moderne, note l'auteur, parce qu'il vit dans l'impossibilité de Dieu, advient à son autonomie et sa responsabilité, sa connaissance du mal et sa culpabilité, sa puissance et sa volonté, mais sa fragilité, aussi bien, et sa solitude. Mais l'essentiel est là, en ce travail de deuil par quoi l'altérité est rapatriée au cœur du psychisme. Un pas de plus, à quoi semble inviter l'argumentaire de l'auteur: l'autre survient au cœur de la psyché comme attestation de la mort de Dieu, comme témoignage d'une absence radicale. Et fondatrice. Il est, écrit Arènes, «transféré dans l'immanence, sans extériorité ni sacralité ». " Mort de Dieu », dès lors, serait un signifiant à vocation universelle, qui dirait en effet toute origine comme rupture.

3 Si la dépossession est bien cette catégorie nucléaire de la mystique dans son incessante quête de Dieu, c'est de se déployer désormais dans le registre propre de l'intériorité, le seul où le sujet est alors capable de Dieu, quand Dieu, en effet, se dépossède. La « fin du religieux " (même si elle ne recouvre pas exactement la fin de la métaphysique ni le « temps » de la mort de Dieu) signe, selon l'auteur, l'entrée en "postmodernité ». Soit. Elle implique plus fondamentalement le dévoilement d'une "subjectivité du croire ", où le « croire » est plus acte performatif, souligne J. Arènes, que fonction relationnelle. La mystique, au-delà des siècles où elle a connu son maximum d'expansion et de tension, se tient au seuil de cette nouvelle aventure de l'esprit, parce que pleinement dans le deuil de dieu. C'est dire sa modernité, et son exigence. L'auteur dit très précisément la conjonction de la mystique avec la « laïcisation de l'espace intérieur ", cet autre nom de la subjectivité venue à sa raison. À sa langue de désir (de Certeau), à sa volonté (Fénelon). Et "à l'archaïsme du monde interne du sujet», que Freud dit le «lieu» où prend sa source la mystique. C'est en cet «archaïsme» que le sujet va mobiliser les conditions de résilience au trauma constitutif du deuil de Dieu. Ainsi du " pur amour ", qui est ce don «sans retour ni calcul», ce consentement à (se) perdre, qui n'est pas pure oblation de soi, mais, si l'on peut dire, ablation absolue, cette énergie en excès d'elle-même qui fait du sujet cela même: personne. Ainsi de l'indifférence, " acte passif » selon François de Sales, mais en quoi se concentrent toutes les violences d'une « âme » en quête d'un dieu « absent ».

4 L'épreuve de la mort de Dieu, ce trauma que l'on peut dire avec Jacques Arènes à l'origine de notre "modernité » occidentale, institue le sujet autonome, libre mais souffrant, souffrant, sans doute, parce que libre. Le défi est alors de "créer du sens à travers la détresse », quand l'événement traumatique fait irruption «dans l'enclos de l'organisme » comme ce que Freud appelle « un corps étranger ». Du sens, c'est-à-dire " ce qui doit advenir », ce qui doit faire gain à travers la perte consommée. Soit, pour le croyant, la foi, cet acte qui devient «l'essence de la vraie religion». Ce «croire performatif », que définit l'auteur dans la filiation de Kierkegaard comme "acte de la foi pure qui se joue au cœur même de l'intimité affective ». Penser Dieu à partir de la mort de Dieu. Penser Dieu à partir du nihilisme. À partir de l'athéisme, que l'auteur entend comme «travail psychique purificateur », dans la mesure où il est " pratique de 
désillusion contre la religiosité du psychisme» - contre le croire comme catégorie "fonctionnelle-naturelle " de la conscience, quand il ne peut être désormais que cet acte assumé en son seul scandale. Retour donc à la mystique, où tout ceci fut expérience vive.

Si la "foi nue », attestation radicale de l'absence, est signe que, le trauma surmonté, une re-naissance peut s'accomplir, on voit que la spiritualité se déploie selon une nouvelle économie. «La foi chrétienne, écrit de Certeau, est expérience de fragilité, moyen de devenir l'hôte d'un autre qui inquiète et fait vivre». Cela valait pour les " saints » du xvii siècle. Cela vaut, a fortiori, pour les spiritualités d'aujourd'hui. Cette fragilité du sujet en l'absence de son dieu, et en présence de cet "autre » qui s'écrit dans l'espace de l'intériorité, se dit dans cette langue dénudée à l'extrême, pour « dire le réel » de l'expérience. Le réel, c'est-à-dire, reprenant les termes d'Arènes, «le pur manque au cœur du sujet ", qu'énoncent aussi bien les états du corps - déchirement, souffrances, "folies", qui sont «paroles sans nom»-, et cette exaltation de toute banalité, cette majoration de la quotidienneté, qui sont témoignages de "pauvreté " comme raison du sujet et matière de son croire. Mais tout ceci, précise l'auteur, exclu de l'ordre de l'image et de l'affect. La mystique comme expérience du désaffect, a-t-on pu dire. Ou, avec Lacan, que l'auteur sollicite ici, champ de la "Chose», cette grammaire des figures de l'altérité, «figures du mal » mais aussi bien «l'Autre absolu du sujet ", et ce "vide au centre du réel » qui soutient en effet le sujet comme sa condition même. Dont témoignent la spiritualité de Marie de La Trinité et "l'épreuve du vide ", entendu comme «mystique du nihilisme actif », cette force de vivre dans un monde "évidé de l'intérieur " - et l'expérience de Thérèse de l'Enfant Jésus, qui «se donne au néant » tout autant que le néant se donne à elle, et qui décide du doute comme protestation de la foi.

6 De Dieu, faire aujourd'hui son deuil. C'est par ce «deuil » seul, propose l'auteur, que le religieux peut faire retour. De l'absence faire une présence renouvelée. Cela s'appelle conversion, cette opération par laquelle «le sujet se donne, ou se laisse donner, un espace de création ». Car la conversion est ce surgissement de la rencontre, aux limites de la vie, entre soi-même et l'autre, que Kierkegaard nomme "acte de foi ", en un temps d'extrême contemporanéité où, d'un coup de force, ou de grâce, se consomme «le rapport du fini et l'infini ». De cet instant où tout bascule, Paul est le nom sur le chemin de Damas. Rien ne revient au même, tout, au contraire, change. Au principe de ce changement, et à la source de cette nouvelle donne du sens, le remaniement des mécanismes archaïques de la conscience, auxquels l'auteur se réfère sous la catégorie analytique de la sublimation, cette " mise au monde de quelque chose de radicalement nouveau ». L'Inconscient est ce " lieu ultradéterminé et complètement insaisissable ", et donc foyer de conservation autant que d'émergence. De «nouveaux espaces intérieurs » s'y inventent, qui ressortirent de ce que Bergson appelait intuition; des énergies nouvelles s'y activent, que Freud nommait travail du négatif. Par ce travail, par cette dynamique, le deuil de Dieu s'ouvre, en suivant l'auteur au plus profond de sa pensée, à un croire libéré de sa dimension référentielle, pour s'affirmer présence ici et maintenant au monde. Croire, dès lors, est affaire profane, et Dieu fragile est aventure humaine en son incertitude. 